\title{
Comparison of exact-exchange calculations for solids in current-spin-density- and
} spin-density-functional theory

Sharma, S.; Pittalis, S.; Kurth, S.; Shallcross, Sam; Dewhurst, J.K.; Gross, E.K.U.

Published in:

Physical Review B Condensed Matter

Link to article, DOI:

10.1103/PhysRevB.76.100401

Publication date:

2007

Document Version

Publisher's PDF, also known as Version of record

Link back to DTU Orbit

Citation (APA):

Sharma, S., Pittalis, S., Kurth, S., Shallcross, S., Dewhurst, J. K., \& Gross, E. K. U. (2007). Comparison of exact-exchange calculations for solids in current-spin-density- and spin-density-functional theory. Physical Review B Condensed Matter, 76(10), 100401. https://doi.org/10.1103/PhysRevB.76.100401

\section{General rights}

Copyright and moral rights for the publications made accessible in the public portal are retained by the authors and/or other copyright owners and it is a condition of accessing publications that users recognise and abide by the legal requirements associated with these rights.

- Users may download and print one copy of any publication from the public portal for the purpose of private study or research.

- You may not further distribute the material or use it for any profit-making activity or commercial gain

- You may freely distribute the URL identifying the publication in the public portal 


\title{
Comparison of exact-exchange calculations for solids in current-spin-density- and spin-density-functional theory
}

\author{
S. Sharma $, 1,2, *$ S. Pittalis, ${ }^{2}$ S. Kurth,${ }^{2}$ S. Shallcross,${ }^{3}$ J. K. Dewhurst, ${ }^{4}$ and E. K. U. Gross ${ }^{2}$ \\ ${ }^{1}$ Fritz, Haber Institute of the Max Planck Society, Faradayweg 4-6, D-14195 Berlin, Germany \\ ${ }^{2}$ Institut für Theoretische Physik, Freie Universität Berlin, Arnimallee 14, D-14195 Berlin, Germany \\ ${ }^{3}$ Department of Physics, Technical University of Denmark, Building 307, DK-2800 Kgs. Lyngby \\ ${ }^{4}$ School of Chemistry, The University of Edinburgh, Edinburgh EH9 3JJ, Scotland
}

(Received 20 March 2007; revised manuscript received 1 June 2007; published 4 September 2007)

\begin{abstract}
The relative merits of current-spin-density- and spin-density-functional theory are investigated for solids treated within the exact-exchange-only approximation. Spin-orbit splittings and orbital magnetic moments are determined at zero external magnetic field. We find that for magnetic ( $\mathrm{Fe}, \mathrm{Co}$, and $\mathrm{Ni}$ ) and nonmagnetic ( $\mathrm{Si}$ and $\mathrm{Ge}$ ) solids, the exact-exchange current-spin-density functional approach does not significantly improve the accuracy of the corresponding spin-density functional results.
\end{abstract}

DOI: 10.1103/PhysRevB.76.100401

PACS number(s): 75.10.Lp, 71.15.Mb, 71.15.Rf

In the past 30 years, several generalizations of density functional theory (DFT) have been proposed. In the early 1970 's, DFT was extended to spin-DFT (SDFT) ${ }^{1}$ by including the spin magnetization as a basic quantity in addition to the density. This allows for coupling of the spin degrees of freedom to external magnetic fields and produces better results for spontaneously spin-polarized systems using approximate functionals. Adding yet another density, the paramagnetic current, leads to the framework of current-SDFT (CSDFT). ${ }^{2,3}$ CSDFT includes the coupling of the external magnetic field, through its corresponding vector potential, to the orbital-degrees of freedom. ${ }^{3}$

SDFT has been enormously successful in predicting the magnetic properties of materials. This success can be attributed to the availability of exchange correlation (XC) functionals which, even though originally designed for nonmagnetic systems, could be systematically extended to the spin polarized case. The most popular of these functionals are the local spin density approximation (LSDA) and the generalized gradient approximation (GGA). CSDFT, on the other hand, has not enjoyed the same attention mainly because of problems which arise in the extension of LSDA and GGA to include the paramagnetic current density. ${ }^{4-6}$ Exposing the homogeneous electron gas to an external magnetic field leads to the appearance of Landau levels which, in turn, give rise to derivative discontinuities in the resulting XC energy density. Using this quantity to construct (semi)local functionals then automatically leads to local discontinuities in the corresponding XC potentials, which are then awkward to use in practical calculations.

Such problems can be avoided with the use of orbital functionals and this fact, coupled with the success of these functionals for SDFT calculations, has led to recent interest in orbital functionals for CSDFT. ${ }^{7-11}$ The results from these works have shown mixed success. A modified version of the original $\mathrm{CSDFT}^{12}$ lead to promising results for spin-orbit induced splittings of bands in solids, such as Si and Ge. ${ }^{9}$ In contrast it was found that for open-shell atoms and quantum dots the difference between SDFT and CSDFT results was minimal. ${ }^{7,8}$ Similarly, calculations for solids using a local vorticity functional ${ }^{13}$ and for quantum dots using a LSDA- type $\mathrm{XC}$ functional ${ }^{14}$ could not establish the superiority of CSDFT over SDFT.

In this work we present a systematic comparison of the relative merits of CSDFT and SDFT for solids. Since the Kohn-Sham (KS) system in CSDFT reproduces the paramagnetic current of the interacting system, one would expect differences between SDFT and CSDFT results for orbital magnetic moments (which can be directly derived from the current). With this in mind we calculate the orbital magnetic moment of the spontaneous magnets $\mathrm{Fe}, \mathrm{Co}$, and Ni. Since CSDFT is believed to improve the spin-orbit induced band splitting in the nonmagnetic semiconductors $\mathrm{Si}$ and $\mathrm{Ge}^{9}$ it makes these materials interesting candidates for a study of the differences between the two approaches.

Following Vignale and Rasolt, ${ }^{2,3}$ the ground-state energy of a (nonrelativistic) system of interacting electrons in the presence of an external magnetic field $\mathbf{B}_{0}(\mathbf{r})=\boldsymbol{\nabla} \times \mathbf{A}_{0}(\mathbf{r})$ can be written as functional of three independent densities: the particle density $\rho(\mathbf{r})$, the magnetization density $\mathbf{m}(\mathbf{r})$, and the paramagnetic current density $\mathbf{j}_{p}(\mathbf{r})$. This functional is given by

$$
\begin{aligned}
E\left[\rho, \mathbf{m}, \mathbf{j}_{p}\right]= & T_{s}\left[\rho, \mathbf{m}, \mathbf{j}_{p}\right]+U[\rho]+E_{\mathrm{XC}}\left[\rho, \mathbf{m}, \mathbf{j}_{p}\right] \\
& +\int \rho(\mathbf{r}) v_{0}(\mathbf{r}) d^{3} r-\int \mathbf{m}(\mathbf{r}) \cdot \mathbf{B}_{0}(\mathbf{r}) d^{3} r \\
& +\frac{1}{c} \int \mathbf{j}_{p}(\mathbf{r}) \cdot \mathbf{A}_{0}(\mathbf{r}) d^{3} r+\frac{1}{2 c^{2}} \int \rho(\mathbf{r}) \mathbf{A}_{0}^{2}(\mathbf{r}) d^{3} r
\end{aligned}
$$

where $T_{s}\left[\rho, \mathbf{m}, \mathbf{j}_{p}\right]$ is the kinetic energy functional of noninteracting electrons, $U[\rho]$ is the Hartree energy, and $E_{\mathrm{XC}}\left[\rho, \mathbf{m}, \mathbf{j}_{p}\right]$ is the exchange-correlation energy. Minimization of Eq. (1) with respect to the three basic densities leads to the Kohn-Sham (KS) equation which reads

$$
\left[\frac{1}{2}\left(-i \boldsymbol{\nabla}+\frac{1}{c} \mathbf{A}_{s}(\mathbf{r})\right)^{2}+v_{s}(\mathbf{r})+\mu_{B} \boldsymbol{\sigma} \cdot \mathbf{B}_{s}(\mathbf{r})\right] \Phi_{j}(\mathbf{r})=\varepsilon_{j} \Phi_{j}(\mathbf{r}) \text {. }
$$

Here $\boldsymbol{\sigma}$ is the vector of Pauli matrices and the $\Phi_{i}$ are spinor valued wave functions. The effective potentials $v_{s}, \mathbf{B}_{s}$, and 
$\mathbf{A}_{s}$ are such that the ground-state densities $\rho, \mathbf{m}$, and $\mathbf{j}_{p}$ of the interacting system are reproduced. These effective potentials are given by

$$
\begin{aligned}
& v_{s}(\mathbf{r})=v_{0}(\mathbf{r})+v_{H}(\mathbf{r})+v_{\mathrm{XC}}(\mathbf{r})+\frac{1}{2 c^{2}}\left[\mathbf{A}_{0}^{2}(\mathbf{r})-\mathbf{A}_{s}^{2}(\mathbf{r})\right], \\
& \mathbf{B}_{s}(\mathbf{r})=\mathbf{B}_{0}(\mathbf{r})+\mathbf{B}_{\mathrm{XC}}(\mathbf{r}), \\
& \mathbf{A}_{s}(\mathbf{r})=\mathbf{A}_{0}(\mathbf{r})+\mathbf{A}_{\mathrm{XC}}(\mathbf{r}) .
\end{aligned}
$$

Here, $v_{0}$ is the external electrostatic potential and $v_{H}(\mathbf{r})$ $=\int \rho\left(\mathbf{r}^{\prime}\right) /\left|\mathbf{r}-\mathbf{r}^{\prime}\right| d^{3} r^{\prime}$ is the Hartree potential. The XC potentials are given as functional derivatives of the XC energy with respect to the corresponding conjugate densities which can be obtained from KS wave functions using the following relations:

$$
\begin{aligned}
& \rho(\mathbf{r})=\sum_{i=1}^{\mathrm{occ}} \Phi_{i}^{\dagger}(\mathbf{r}) \Phi_{i}(\mathbf{r}), \\
& \mathbf{m}(\mathbf{r})=-\mu_{B} \sum_{i=1}^{\mathrm{occ}} \Phi_{i}^{\dagger}(\mathbf{r}) \boldsymbol{\sigma} \Phi_{i}(\mathbf{r}), \\
& \mathbf{j}_{p}(\mathbf{r})=\frac{1}{2 i} \sum_{i=1}^{\mathrm{occ}}\left\{\Phi_{i}^{\dagger}(\mathbf{r}) \nabla \Phi_{i}(\mathbf{r})-\left[\nabla \Phi_{i}^{\dagger}(\mathbf{r})\right] \Phi_{i}(\mathbf{r})\right\},
\end{aligned}
$$

where the sum runs over the occupied orbitals. For practical calculations, an approximation for the XC energy functional $E_{\mathrm{XC}}\left[\rho, \mathbf{m}, \mathbf{j}_{p}\right]$ has to be adopted. Here we concentrate on approximations of the XC functional which explicitly depend on the KS orbitals and therefore only implicitly on the densities. Such orbital functionals are usually treated within the framework of the so-called optimized effective potential (OEP) method ${ }^{15-18}$ where the XC potential is obtained as solution of the OEP integral equation. Recently, the OEP method has been generalized to noncollinear SDFT $^{19}$ and CSDFT. $^{7,8}$ Another generalization of the OEP method in the context of a spin-current DFT (SCDFT) based on a different choice of densities has also been put forward. ${ }^{11}$ In the present work the formalism of Refs. 7 and 8 is used and the corresponding OEP equations can be put in a compact form as

$$
\begin{array}{r}
\sum_{k=1}^{\text {occ }} \Phi_{k}^{\dagger}(\mathbf{r}) \Psi_{k}(\mathbf{r})+\text { h.c. }=0 \\
-\mu_{B} \sum_{k}^{\text {occ }} \Phi_{k}^{\dagger}(\mathbf{r}) \boldsymbol{\sigma} \Psi_{k}(\mathbf{r})+\text { h.c. }=0,
\end{array}
$$$$
\frac{1}{2 i} \sum_{k}^{\mathrm{occ}}\left\{\Phi_{k}^{\dagger}(\mathbf{r}) \nabla \Psi_{k}(\mathbf{r})-\left[\nabla \Phi_{k}^{\dagger}(\mathbf{r})\right] \Psi_{k}(\mathbf{r})\right\}+\text { h.c. }=0,
$$

where the so-called orbital shifts ${ }^{17,20}$ are defined as $\Psi_{k}(\mathbf{r})$ $=\sum_{j}^{\text {unocc }} \Phi_{j}(\mathbf{r}) \Lambda_{k j} / \varepsilon_{k}-\varepsilon_{j}$, here the summation runs over the unoccupied states and

$$
\begin{aligned}
\Lambda_{k j}= & \int \mathrm{d}^{3} r^{\prime}\left(v_{\mathrm{XC}}\left(\mathbf{r}^{\prime}\right) \rho_{k j}\left(\mathbf{r}^{\prime}\right)+\frac{1}{c} \mathbf{A}_{\mathrm{XC}}\left(\mathbf{r}^{\prime}\right) \cdot \mathbf{j}_{p k j}\left(\mathbf{r}^{\prime}\right)\right. \\
& \left.-\mathbf{B}_{\mathrm{XC}}\left(\mathbf{r}^{\prime}\right) \cdot \mathbf{m}_{k j}\left(\mathbf{r}^{\prime}\right)-\Phi_{j}^{\dagger}\left(\mathbf{r}^{\prime}\right) \frac{\delta E_{\mathrm{XC}}}{\delta \Phi_{k}^{\dagger}\left(\mathbf{r}^{\prime}\right)}\right),
\end{aligned}
$$

where $\rho_{k j}(\mathbf{r})=\Phi_{j}^{\dagger}(\mathbf{r}) \Phi_{k}(\mathbf{r}), \quad \mathbf{m}_{k j}(\mathbf{r})=-\mu_{B} \Phi_{j}^{\dagger}(\mathbf{r}) \boldsymbol{\sigma} \Phi_{k}(\mathbf{r})$, and $\mathbf{j}_{p k j}(\mathbf{r})=1 / 2 i\left\{\Phi_{j}^{\dagger}(\mathbf{r}) \nabla \Phi_{k}(\mathbf{r})-\left[\boldsymbol{\nabla} \Phi_{j}^{\dagger}(\mathbf{r})\right] \Phi_{k}(\mathbf{r})\right\}$.

Equation (5) has a structure very similar to the OEP equations for noncollinear SDFT differing only in the definition of the matrix $\Lambda$, which now also contains an extra term depending upon the current density and its conjugate field. Due to their similar structure the CSDFT OEP equations are solved by generalizing the "residue algorithm," successfully applied to solve the noncollinear SDFT equations. ${ }^{19-21}$ The only difference in the case of CSDFT is the introduction of an additional residue coming from the third OEP equation in Eq. (5). In the present work we have used the exchange-only exact-exchange (EXX) functional to solve the OEP equations. The (gauge invariant) EXX energy functional is the Fock exchange energy but evaluated with KS spinors

$$
E_{x}^{\operatorname{EXX}}\left[\left\{\Phi_{i}\right\}\right] \equiv-\frac{1}{2} \iint \sum_{i, j}^{\mathrm{occ}} \frac{\Phi_{i}^{\dagger}(\mathbf{r}) \Phi_{j}(\mathbf{r}) \Phi_{j}^{\dagger}\left(\mathbf{r}^{\prime}\right) \Phi_{i}\left(\mathbf{r}^{\prime}\right)}{\left|\mathbf{r}-\mathbf{r}^{\prime}\right|} d^{3} r d^{3} r^{\prime} .
$$

In order to keep the numerical analysis as accurate as possible, in the present work all calculations are performed using the state-of-the-art full-potential linearized augmented plane wave (FPLAPW) method, ${ }^{22}$ implemented within the EXCITING code. ${ }^{23}$ The single-electron problem is solved using an augmented plane wave basis without using any shape approximation for the effective potential. Likewise, the magnetization and current densities and their conjugate fields are all treated as unconstrained vector fields throughout space. The deep lying core states (3 Ha below the Fermi level) are treated as Dirac spinors and valence states as Pauli spinors. To obtain the Pauli spinor states, the Hamiltonian containing only the scalar fields is diagonalized in the LAPW basis: this is the first-variational step. The scalar states thus obtained are then used as a basis to set up a second-variational Hamiltonian with spinor degrees of freedom, which consists of the first-variational eigenvalues along the diagonal, and the matrix elements obtained from the external and effective vector fields in Eq. (2). This is more efficient than simply using spinor LAPW functions, but care must be taken to ensure there are a sufficient number of first-variational eigenstates for convergence of the second-variational problem. Spinorbit coupling is also included at this stage.

As shown above for CSDFT, the magnetic field couples not only to spin but also to the orbital degrees of freedom through the vector potential. This makes CSDFT specifically important for magnetic materials and particularly interesting for their orbital properties. By analogy with SDFT, one might expect that the introduction of the paramagnetic current density gives an improvement in properties such as orbital moments and spin-orbit induced band splitting, which are related to this new basic variable. However, within the framework of existing functionals it is yet to be established 
TABLE I. Orbital magnetic moments for bulk Fe, Co, and $\mathrm{Ni}$ in $\mu_{B}$. The experimental data are taken from Ref. 24. The final row lists the average percentage deviation of the numerical results from the experimental value.

\begin{tabular}{lccccc}
\hline \hline Solid & Exp. & LSDA & $\begin{array}{r}\text { SDFT } \\
\text { GGA }\end{array}$ & EXX & $\begin{array}{c}\text { CSDFT } \\
\text { EXX }\end{array}$ \\
\hline Fe & 0.08 & 0.053 & 0.051 & 0.034 & 0.034 \\
Co & 0.14 & 0.069 & 0.073 & 0.013 & 0.013 \\
$\mathrm{Ni}$ & 0.05 & 0.038 & 0.037 & 0.029 & 0.029 \\
& & 36.2 & 36.7 & 63.4 & 63.4 \\
\hline
\end{tabular}

conclusively that CSDFT performs better than SDFT for these properties. The recent development of the OEP method both for SDFT and CSDFT allows for a direct comparison of these two approaches for the same $\mathrm{XC}$ functional, namely, EXX.

The orbital moments of spontaneous magnets $\mathrm{Fe}, \mathrm{Co}$, and $\mathrm{Ni}$, in the absence of external magnetic fields and with spinorbit coupling included, are presented in Table I. For SDFT, the LSDA, GGA, and EXX functionals are used, while for CSDFT the values are obtained using the EXX functional. It is clear from Table I that there is no difference between the results obtained using EXX-CSDFT and EXX-SDFT. Formally, the $\mathbf{j}_{p}$ determined from SDFT does not correspond to the true paramagnetic current density of the fully interacting system. Nevertheless, it is standard practice to compute the orbital magnetic moment $\mathbf{L}$, similar to those listed in Table I, which is related to $\mathbf{j}_{p}$ from the $\mathrm{KS}$ orbitals by the relation $\mathbf{L}=1 / 2 \int \mathbf{r} \times \mathbf{j}_{p}(\mathbf{r}) d^{3} r$. The fact the EXX-SDFT and EXXCSDFT orbital moments are so close may be viewed as a post hoc justification of this practice for magnetic metals. It should also be noted that in comparison to experiments the EXX results are significantly worse than their LSDA and GGA counterparts. One reason, of course, is the fact that LSDA and GGA also include correlation in an approximate way which is neglected completely within the EXX framework.

In a recent work ${ }^{9}$ it is shown that the use of the EXX functional in the framework of SCDFT, improves the spinorbit induced splitting of the bands in semiconductors. Unfortunately, it is not clear if this improvement is due to the use of different functionals (going from LSDA to EXX), or due to the use of an extra density when going from SDFT to

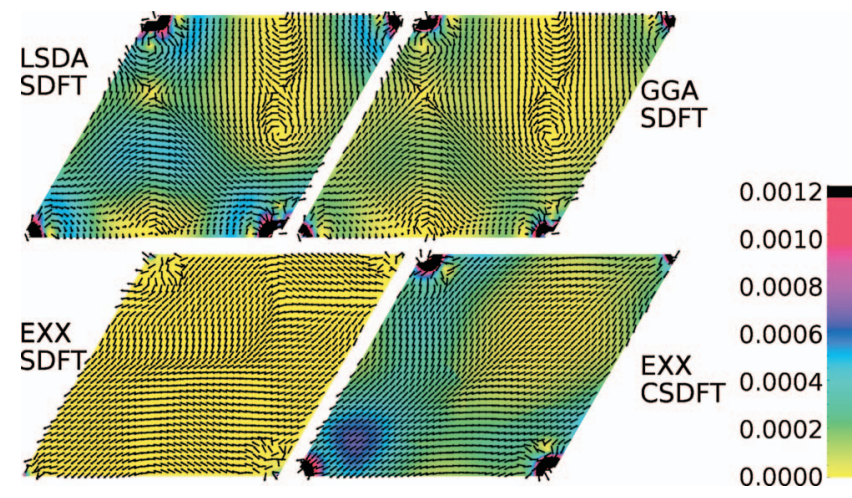

FIG. 1. (Color) Paramagnetic current density for $\mathrm{Ge}$, in the [110] plane, calculated using the SDFT and CSDFT. Arrows indicate the direction and information about the magnitude (in atomic units) is given in the color bar.

CSDFT. This has motivated us to compare CSDFT and SDFT results for this quantity using the same functional in both cases. We have determined the value of this splitting for solid $\mathrm{Si}$ and $\mathrm{Ge}$ and the results are presented in Table II. While the EXX functional significantly improves the agreement with experimental values, there is almost no change on going from SDFT to CSDFT. Thus the improvement is solely due to the orbital based functional. We also note that the EXX-CSDFT results of Ref. 9 are significantly different from ours, and in much worse agreement with experiments. This might be due to the use of pseudopotentials in the previous work. In this respect it is worth noting that EXX derived KS energy gaps also show significant differences depending on whether an all-electron full-potential or pseudopotential method is used. ${ }^{25}$

The paramagnetic current density of Ge for LSDA, GGA, EXX-SDFT, and EXX-CSDFT is plotted in Fig. 1. Ge is chosen as an example since the spin-orbit induced splitting is largest for this system and, unlike in the case of metallic orbital moments, this quantity does show some difference on going from SDFT to CSDFT. We immediately notice that there is no significant qualitative difference between the LSDA and GGA currents. There are, however, pronounced differences in the current density between LSDA/GGA and EXX-(C)SDFT: the current in the latter case being smaller and more homogeneous than that of the former. This is an interesting finding since it indicates the tendency of (semi)local functionals towards higher values of the paramagnetic current density. It is worthwhile noting previous EXX-

TABLE II. Spin-orbit induced splittings for bulk Ge and $\mathrm{Si}$ in meV. The experimental data is taken from Ref. 26. The final row lists the average percentage deviation of numerical results from the experimental value.

\begin{tabular}{lcccccc}
\hline \hline $\begin{array}{l}\text { Symmetry } \\
\text { point }\end{array}$ & Exp. & LSDA & GGA & EXX & EXX (present) & EXX (Ref. 9) \\
\hline Ge $\Gamma_{7 v-8 v}$ & 297 & 311 & 296 & 291.3 & 289 & 258.1 \\
Ge $\Gamma_{6 c-8 c}$ & 200 & 229.7 & 220 & 201.3 & 199 & 173.3 \\
Si $\Gamma_{25 v}$ & 44 & 50 & 58 & 42.5 & 45.5 & 42.5 \\
& & 9.5 & 14.0 & 2.0 & 2.2 & 10.5 \\
\hline \hline
\end{tabular}


(C)SDFT results for open-shell atoms ${ }^{7}$ where this effect is even more pronounced and leads to vanishing currents.

Even though the EXX-SDFT current is considerably lower in magnitude than that of EXX-CSDFT and also has a less symmetric structure, the spin-orbit splittings for the two cases are almost the same. Similar conclusions regarding the total energies were also drawn for quantum dots in external magnetic fields studied using $\mathrm{EXX}^{8}$ and other functionals of the current density. ${ }^{14}$ From Fig. 1 it is also clear that one of the major effects of using the OEP method and of using $\mathbf{j}_{p}$ as an extra density is to change the local structure of the paramagnetic current, which in turn suggests that quantities depending on local properties of the currents, such as chemical shifts, might exhibit larger differences in the two approaches. Such calculations ${ }^{27}$ of chemical shifts, performed using local functionals, found that for molecules this is not the case. The effect of the EXX functional on these shifts may be an interesting subject for future investigations.

To summarize, in this work we have presented EXXSDFT and CSDFT calculations for solids. The orbital magnetic moments of $\mathrm{Fe}, \mathrm{Co}$, and $\mathrm{Ni}$ and the spin-orbit induced band splitting of $\mathrm{Si}$ and $\mathrm{Ge}$ are computed. Our analysis shows only minor differences between EXX-CSDFT and SDFT results. The spin-orbit induced band splittings in EXX calculations are in rather good agreement with experiments, while the results for the orbital moments are worse than the LSDA or GGA values. This highlights the importance of a proper treatment of correlations for the accurate determination of the orbital moments.

We acknowledge Deutsche Forschungsgemeinschaft and NoE NANOQUANTA Network (Grant No. NMP4-CT-200450019) for financial support. *sangeeta.sharma@physik.fu-berlin.de

${ }^{1}$ U. von Barth and L. Hedin, J. Phys. C 5, 1629 (1972).

${ }^{2}$ G. Vignale and M. Rasolt, Phys. Rev. Lett. 59, 2360 (1987).

${ }^{3}$ G. Vignale and M. Rasolt, Phys. Rev. B 37, 10685 (1988).

${ }^{4}$ P. Skudlarski and G. Vignale, Phys. Rev. B 48, 8547 (1993).

${ }^{5}$ Y. Takada and H. Goto, J. Phys.: Condens. Matter 10, 11315 (1998).

${ }^{6}$ K. Higuchi and M. Higuchi, Phys. Rev. B 74, 195122 (2006).

${ }^{7}$ S. Pittalis, S. Kurth, N. Helbig, and E. K. U. Gross, Phys. Rev. A 74, 062511 (2006).

${ }^{8}$ N. Helbig, S. Kurth, S. Pittalis, E. Räsänen, and E. K. U. Gross, arXiv:cond-mat/0605599 (unpublished).

${ }^{9}$ S. Rohra, E. Engel, and A. Görling, arXiv:cond-mat/0608505 (unpublished).

${ }^{10}$ T. Heaton-Burgess, P. Ayers, and W. Yang, Phys. Rev. Lett. 98, 036403 (2007).

${ }^{11}$ S. Rohra and A. Görling, Phys. Rev. Lett. 97, 013005 (2006).

${ }^{12}$ K. Bencheikh, J. Phys. A 36, 11929 (2003).

${ }^{13}$ H. Ebert, M. Battocletti, and E. K. U. Gross, Europhys. Lett. 40, 545 (1997).

${ }^{14}$ H. Saarikoski, E. Räsänen, S. Siljamäki, A. Harju, M. J. Puska, and R. M. Nieminen, Phys. Rev. B 67, 205327 (2003).

${ }^{15}$ R. Sharp and G. Horton, Phys. Rev. 90, 317 (1953).

${ }^{16}$ J. D. Talman and W. F. Shadwick, Phys. Rev. A 14, 36 (1976).
${ }^{17}$ T. Grabo, T. Kreibich, S. Kurth, and E. K. U. Gross, in Strong Coulomb Correlations in Electronic Structure Calculations: Beyond Local Density Approximations, edited by V. Anisimov (Gordon and Breach, Amsterdam, 2000), p. 203.

${ }^{18}$ E. Engel and S. H. Vosko, Phys. Rev. A 47, 2800 (1993).

${ }^{19}$ S. Sharma, J. K. Dewhurst, C. Ambrosch-Draxl, S. Kurth, N. Helbig, S. Pittalis, S. Shallcross, L. Nordström, and E. K. U. Gross, Phys. Rev. Lett. 98, 196405 (2007).

${ }^{20}$ S. Kümmel and J. P. Perdew, Phys. Rev. Lett. 90, 043004 (2003).

${ }^{21}$ S. Kümmel and J. P. Perdew, Phys. Rev. B 68, 035103 (2003).

${ }^{22}$ D. J. Singh, in Planewaves, Pseudopotentials and the LAPW Method (Kluwer, Dordrecht, 1994).

${ }^{23}$ J. K. Dewhurst, S. Sharma, and C. Ambrosch-Draxl, http:// exciting.sourceforge.net/

${ }^{24}$ M. B. Stearns, in Magnetic Properties of $3 d, 4 d$, and $5 d$ Elements, Alloys and Compounds, edited by Landolt-Boernstein (Springer, Berlin, 1987), Vol. III/19a.

${ }^{25}$ S. Sharma, J. K. Dewhurst, and C. Ambrosch-Draxl, Phys. Rev. Lett. 95, 136402 (2005).

${ }^{26}$ O. Madelung, Semiconductors: Data Handbook (Springer-Verlag, Berlin, 2004).

${ }^{27}$ A. M. Lee, N. C. Handy, and S. M. Colwell, J. Chem. Phys. 103, 10095 (1995). 